\title{
Effect of Cardiovascular Training on Functional Capacity in Post-Acute Rehabilitation of COVID-19 Patients
}

\author{
Dragana Dragičević-Cvjetković, ${ }^{1,2}$ Drinka Stevandić
}

\begin{abstract}
Introduction: In the overall strategy of developing the optimal treatment of patients after COVID-19 infection, recommended by the World Health Organization, rehabilitation plays one of the key roles in improving the functional capacity of these patients and thus their quality of life. The aim of this study was to investigate the effect of cardiovascular training during post-acute rehabilitation on the functional capacity of patients after moderate COVID-19 pneumonia. Methods: The prospective study included 84 patients of both sexes, mean age $57.92 \pm 11.79$ years, who were hospitalised at the Institute of Physical Medicine and Rehabilitation "Dr Miroslav Zotović" Banja Luka due to moderate pneumonia caused by the COVID-19 virus and after they finished acute rehabilitation. All patients underwent cardiovascular training three times per week and occupational therapy during 28 days of stationary post-acute rehabilitation. The follow-up parameter was a six-minute walk (6-MWT) test at admission and discharge from post-acute rehabilitation. Student t-test for paired samples was used for statistical analysis, and the value of $p<0.05$ was taken as statistical significance.

Results: The functional capacity of the cardiovascular and respiratory system was statistically significantly improved at discharge compared to admission $(\mathrm{p}<0.05)$.

Conclusion: Targeted cardiovascular training during post-acute rehabilitation leads to improvement of functional capacities of patients after moderate COVID-19 pneumonia.
\end{abstract}

Key words: Cardiovascular training; Post-acute rehabilitation; COVID-19.
(1) Institute of Physical Medicine and Rehabilitation "Dr Miroslav Zotovic", Banja Luka, the Republic of Srpska, Bosnia and Herzegovina.

(2) Faculty of Medicine, University of Banja Luka, the Republic of Srpska, Bosnia and Herzegovina.

Correspondence: DRAGANA

DRAGIČEVIĆ-CVJETKOVIĆ E: dragicevicdr@gmail.com T: +38765711138

\section{ARTICLE INFO}

Received: 16 August 2021 Revision received: 18 September 2021 Accepted: 18 September 2021

\section{Introduction}

Conducting rehabilitation during the COVID-19 pandemic is a major challenge. On the one hand, the need for rehabilitation has increased, but the way of organisation and timing of its implementation required the reorganisation of rehabilitation systems around the world. ${ }^{1,2}$ The World Health Organization (WHO) has integrated rehabilitation as one of the key links in the chain of the overall treatment of COVID-19 patients, whether it is acute or post-acute rehabilitation. ${ }^{3}$ The main goals of post-acute rehabilitation of these patients are reduction of current problems, training in breathing techniques and improvement of lung function, improvement of general fitness status, and ultimately improvement of quality of life. A 6-MWT was used as a test to assess the effect 
of post-acute rehabilitation. This test was introduced in 2020 according to the recommendations of the American Thoracic Association as a test to assess aerobic capacity and endurance. ${ }^{4}$ However, this test can also be used to assess functional capacity in amputees, patients with neurological and muscular diseases. ${ }^{5}$

The aim of this study was to investigate the effect of cardiovascular training in post-acute rehabilitation on the functional capacity of patients after moderate COVID-19 pneumonia.

\section{Methods}

A prospective study followed 84 patients who, after hospitalisation due to moderate pneumonia caused by the COVID-19 virus and finished acute rehabilitation, underwent post-acute rehabilitation at the Institute for Physical Medicine and Rehabilitation "Dr Miroslav Zotović" Banja Luka in period from October 2020 to March 2021. Criteria for admission to post-acute rehabilitation was inpatient treatment of patients with COVID-19 pneumonia. Criteria for inclusion in the study were hospitalised patients of both sexes with moderate pneumonia caused by COVID-19 virus with no other comorbidities except hypertension. The exclusion criteria were patients younger than 30 years and patients with associated chronic diseases except hypertension. All patients underwent cardiovascular training three times per week and occupational therapy during 28 days of inpatient post-acute rehabilitation. Cardiovascular training included the implementation of the following rehabilitation interventions: breathing gymnastics, muscle strength training, endurance training, ergometer, back gymnastics, pelvic floor muscle training. Through the modalities of occupational therapy, patients underwent progressive muscle relaxation, practicing daily life activities, and educating patients about self-help measures. Patients were tested using 6-MWT on admission and discharge from post-acute rehabilitation. The walk distance as a result of test used for evaluation of cardio-respiratory capacity. Student t-test for paired samples was used for statistical analysis, and the value $\mathrm{p}<0.05$ was taken as statistical significance.

\section{Results}

The investigated sample of patients consisted of 84 patients, with a mean age of $57.92 \pm 11.79$ years (Figure 1, Figure 2).

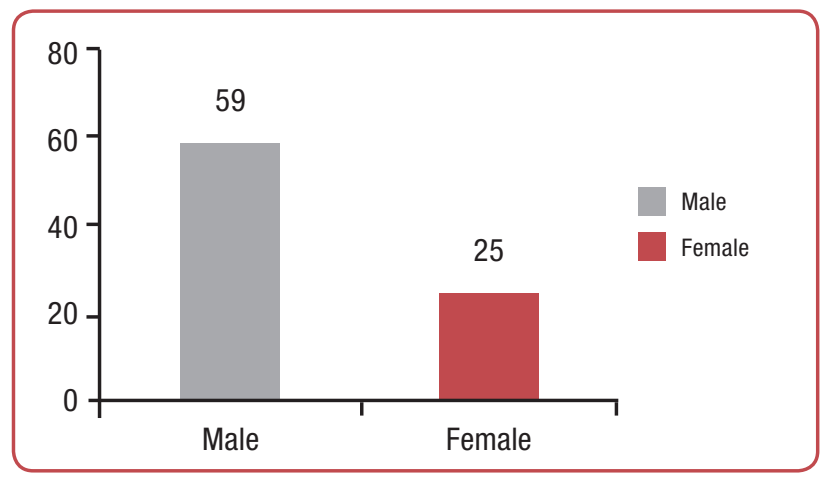

Figure 1: Distribution of patients by gender

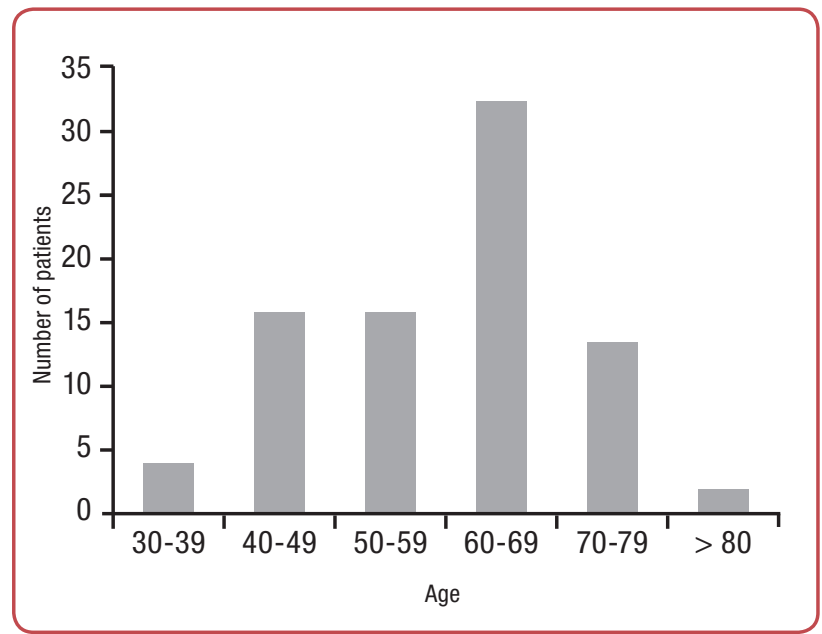

Figure 2: Distribution of patients by age

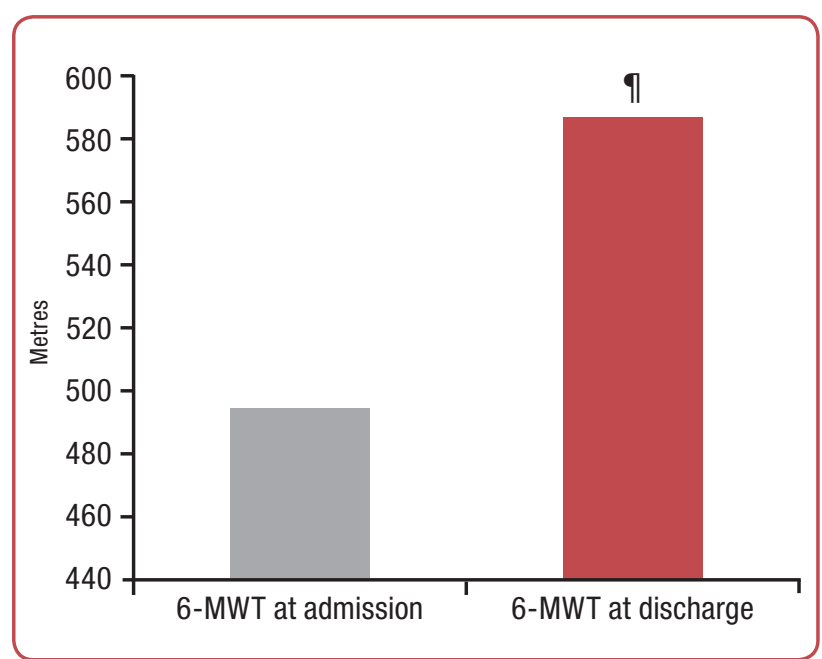

Figure 3: Mean value 6-minute walk test (6-MWT) in postCOVID-19 patients at admission and discharge from post-acute rehabilitation (metres)

If $p<0.05$ 
The average time from completion of primary hospital treatment and acute rehabilitation to admission to post-acute rehabilitation was 50 days.

The values of the 6-MWT on admission and discharge from post-acute rehabilitation were taken as average values. At admission, the average value of the 6-MWT was $493 \mathrm{~m}$. At discharge, the value of the monitored parameter was statistically significantly better at $583 \mathrm{~m}(\mathrm{p}<0.05)$ (Figure 3).

\section{Discussion}

This study was based on the evaluation of the effects of the applied modalities of post-acute rehabilitation in patients suffering from moderate pneumonia caused by the COVID-19. Since most patients complain of fatigue, shortness of breath, palpitations, and loss of muscle mass after suffering from COVID-19 pneumonia, the goal of this rehabilitation is to minimise these symptoms. Rehabilitation was performed in accordance with the basic principles of cardiovascular and respiratory rehabilitation. ${ }^{5}$ The average time from completion of primary hospital treatment and acute rehabilitation to admission to post-acute rehabilitation was 50 days. Patients underwent cardiovascular training three times per week with occupational therapy for a total of 28 days. A 6-MWT as a parameter to assess the effect of the applied therapy was used. Positive effects of applied aerobic training in patients on functional cardio-respiratory capacity were observed, which was shown in a statistically significant improvement in the average value of the 6-MWT on discharge from post-acute COVID-19 rehabilitation. During the post-acute rehabilitation, side effects of the applied therapeutic interventions were not determined.

Although these are the challenges of the "new disease" there are many studies that testify to the benefits of cardiovascular training on the financial capacity of patients after COVID-19 pneumonia. ${ }^{6-13}$ These positive effects can be explained by understanding the mechanisms of action of applied therapeutic interventions in the plan of cardio-respiratory rehabilitation. Cardiovascular training improves the functional capacity of patients after COVID-19 pneumonia in several ways. By improving the strength and endurance of the respiratory muscles, additional ventilation is improved. Combining aerobic capacity reduces free radical production and oxidative damage, reduces cough and improves airway clearance. Conducting aerobic training improves the immune response. ${ }^{14-17}$

One of the limiting factors is the short follow-up period, although there are studies that report positive short-term effects of applied cardiovascular training in post-COVID-19 patients. ${ }^{8-11}$

Besides, the period of time between the end of acute rehabilitation and starting post-acute rehabilitation is a parameter that should be considered in the future. This is especially important from the aspect of the found average values of the 6-MWT at admission. In this study, the average values of this parameter were in the range recommended for the age of 20-50 years. ${ }^{4}$ If patients were admitted earlier for post-acute rehabilitation, the effect could be even better and clinically more significant. Of course, it is indisputable that the criteria for admission to post-acute rehabilitation are primarily met. This means it is necessary to conduct the post-acute rehabilitation at the right time and with the right effect, without side effects and complications, that criteria for admission of patients must be clearly defined and fulfilled. Surely this will happen in the future connectivity of scientific truth in the field of treatment and rehabilitation of patients after COVID-19 pneumonia. The criteria will be changing and supplementing within the overall management of these patients and improve their functional capacity and quality of life.

\section{Conclusion}

The positive effects of cardiovascular training were verified in patients after post-acute rehabilitation after COVID-19 moderate pneumonia in the short-term follow-up period. It is indisputable that that this is a dynamic clinical and scientific field and that in the future numerous reports will define the optimal components of post-acute rehabilitation, the dynamics of its implementation, and tools for monitoring its outcome. 


\section{Acknowledgements}

None.

\section{Conflict of interest}

None.

\section{References}

1. Saverino A, Baiardi P, Galata G, Pedemonte G, Vassallo C, Pistarini C. The challenge of reorganizing rehabilitation services at the time COVID-19 pandemic: a new digital and artificial intelligence platform to support team work in planning and delivering safe and high quality care. Front Neurol 2021 Apr 29;12:643251. doi: 10.3389/ fneur.2021.643251.

2. Andrenelli E, Negrini F, de Sire A, Arienti C, Patrini M, Negrini S, Ceravolo M. Systematic rapid living review on rehabilitation needs due to COVID-19: update to May 31st, 2020. Eur J Phys Rehabilit Med 2020;56(3):347-53.

3. Pan American Health Organisation (PAHO), World Health Organisation (WHO). Rehabilitation considerations during the COVID-19 outbreak [Internet]. [Cited 1 Sep 2021]. Available from: https://iris.paho.org/handle/10665.2/51979.

4. Rossini D, Venturini E, Barni L, Dardi O, Bonaguidi E, P Cavicchioli, et al. Comparison between the 6-minute walking test (6MWT) and gait speed (GS) during outpatient cardiac rehabilitation. ESC Preventive Cardiology 2021;28(1):307-11.

5. Du H, Davidson PM, Everett B, Salamonson Y, Zecchin R, Rolley JX, et al. Correlation between a self-administered walk test and a standardised Six Minute Walk Test in adults. Nurs Health Sci 2011;13(2):114-7.

6. Zampogna E, Paneroni M, Belli S, Aliani M, Gandolfo A, Visca D, et al. Pulmonary rehabilitation in patients recovering from COVID-19. Respiration 2021;100:416-22.

7. Spielmanns M, Pekacka-Egli AM, Schoendorf S, Windisch W, Hermann M. Effects of a comprehensive pulmonary rehabilitation in severe post-COVID-19 patients. Int J Environ Res Public Health 2021;18:2695-708.

8. Tozato C, Costa Ferreira BF, Pereira Dalavina J, Vitelli Molinari C, dos Santos Alves VL. Cardiopulmonary rehabilitation in post-COVID-19 patients: case series. Rev Bras Ter Intensiva 2021;33(1):167-71.

9. Yan H, Ouyang Y, Wang L, Luo X, Zhan Q. Effect of respiratory rehabilitation training on elderly patients with
COVID-19: A protocol for systematic review and meta-analysis. Medicine (Baltimore) 2020 Sep 11;99(37):e22109. doi: 10.1097/MD.0000000000022109.

10. Sun T, Guo L, Tian F, Dai T, Xing X, Zhao J, Li Q. Rehabilitation of patients with COVID-19. Expert Rev Respir Med 2020 Dec;14(12):1249-56.

11. Carneiro I, Costa AJ, Lima A, Machado Vaz I. Protocol proposal to the initial assessment of the post-intensive care syndrome in COVID-19 patients, in an inpatient rehabilitation setting. Rehabilitacion (Madr) 2021 Jan-Mar;55(1):82. doi: 10.1016/j.rh.2020.09.003.

12. Larina V, Karpenko D, Soloviyev S, Sheregova E. Rehabilitation of the elderly patients after pneumonia caused by SARS-COV-2: the continuity of inpatient and outpatient stages. Russian Journal of Geriatric Medicine 2021;(4):327-35.

13. Solon K, Larson A, Ronnebaum J, Stevermer C. Rehabilitation outcomes for patients with severe presentation of COVID-19: A case series. J Acute Care Phys Ther 2021 Jul;12(3):115-21.

14. Malcolm MP. Occupational therapy in postacute care for survivors of COVID-19: research gaps we need to fill. Am J Occup Ther 2021 Jul-Aug;75(Supplement_1):7511347020p1-7511347020p5. doi: 10.5014/ ajot.2021.049195.

15. Baumann FT, Zimmer P, Finkenberg K, Hallek M, Bloch $\mathrm{W}$, Elter T. Influence of endurance exercise on the risk of pneumonia and Fever in leukemia and lymphoma patients undergoing high dose chemotherapy. A pilot study. J Sports Sci Med 2012 Dec 1;11(4):638-42.

16. Toledo AC, Magalhaes RM, Hizume DC, Vieira RP, Biselli PJ, Moriya HT, et al. Aerobic exercise attenuates pulmonary injury induced by exposure to cigarette smoke. Eur Respir J 2012 Feb;39(2):254-64.

17. Vieira RP, Toledo AC, Ferreira SC, Santos AB, Medeiros MC, Hage $M$, et al. Airway epithelium mediates the anti-inflammatory effects of exercise on asthma. Respir Physiol Neurobiol 2011 Mar 15;175(3):383-9. 